\title{
Statistics of Tunneling Events in Three-Terminal Hybrid Devices with Quantum Dot
}

\author{
G. MichaŁeK $^{a, *}$, B.R. BuŁKA ${ }^{a}$, T. Domański ${ }^{b}$, K.I. Wysokiński ${ }^{b}$ \\ ${ }^{a}$ Institute of Molecular Physics, Polish Academy of Sciences, ul. M. Smoluchowskiego 17, 60-179 Poznań, Poland \\ ${ }^{b}$ Institute of Physics, M. Curie-Skłodowska University, pl. M. Curie-Skłodowskiej 1, 20-031 Lublin, Poland
}

\begin{abstract}
We investigate statistics of the tunneling events in the short time limit in terms of the waiting time distribution (WTD), defined as the probability for a delay time between two subsequent transitions of particles, and consider it for a quantum dot (QD) strongly coupled to a superconducting and weakly coupled to two normal electrodes. Our study focuses on the WTD in the subgap transport, when coherent exchange of the Cooper pairs occurs between the QD and the superconductor. The dynamics can be described in terms of a Markovian generalized master equation for the reduced density matrix. We observe coherent oscillations between the Andreev bound states in the correlated jumps, both for the local and non-local WTDs. In addition the analysis of the transient currents give us some insight into dominant relaxation processes in short time scales.
\end{abstract}

DOI: 10.12693/APhysPolA.133.391

PACS/topics: 74.45.+c, 73.23.Hk, 72.70.+m, 73.63.Kv

\section{Introduction}

To fully characterize charge current fluctuations in mesoscopic systems it is necessary to study statistics of tunneling events, e.g. by means of the full counting statistics (FCS) or the waiting time distribution (WTD) in the long or the short time limit, respectively. WTDs in the mesoscopic systems have been studied mainly in two-terminal devices [1]. Particulary interesting is an analysis of the WTD in systems with superconducting correlations where coherent oscillations of Cooper pairs between QD states show interesting dynamics [2]. Such high frequency charge dynamics can be probed in experiments performed in multiterminal hybrid devices consisting of the QD coupled to one superconducting and several normal electrodes [3].

\section{Method}

In the paper we analyze dynamics of tunneling electrons in the hybrid system which consists of the quantum dot (QD) strongly coupled to the superconducting electrode and weakly coupled to two normal metal electrodes. We are interested in a subgap transport (for large superconducting gap $\Delta \rightarrow \infty$ ) where an effective Hamiltonian for the QD in proximity to the superconducting electrode in the BCS approximation takes the form $H_{\text {eff }}=\epsilon_{0} \sum_{\sigma} d_{\sigma}^{\dagger} d_{\sigma}+U n_{\uparrow} n_{\downarrow}-\Gamma_{S}\left(d_{\uparrow}^{\dagger} d_{\downarrow}^{\dagger}+d_{\downarrow} d_{\uparrow}\right) / 2$, where $\epsilon_{0}$ is the single particle energy, $d_{\sigma}^{\dagger}\left(d_{\sigma}\right)$ create (annihilate) an electron with spin $\sigma=\{\uparrow, \downarrow\}$ on QD, $n_{\sigma} \equiv d_{\sigma}^{\dagger} d_{\sigma}$ is the number operator, $U$ denotes a Coulomb interaction on QD and $\Gamma_{S}$ describes coupling between the QD

*corresponding author; e-mail: grzechal@ifmpan.poznan.pl and the superconducting electrode. The effective Hamiltonian $H_{\text {eff }}$ mixes the empty $|0\rangle$ and double occupied $|D\rangle \equiv|\uparrow \downarrow\rangle$ states on QD. It has four eigenvalues: two singly occupied (odd) QD states $|\uparrow\rangle$ and $|\downarrow\rangle$ with energy $\epsilon_{0}$ and two states with even number of electrons $| \pm\rangle=\alpha_{ \pm}|0\rangle \mp \alpha_{\mp}|D\rangle$, where $\alpha_{ \pm}=\sqrt{1 / 2 \mp \delta /\left(4 \epsilon_{A}\right)}$ with energies $\epsilon_{+}=\delta / 2 \pm \epsilon_{A}$. The excitation energies of QD define Andreev bound states $E_{\gamma \gamma^{\prime}}^{A}=\gamma U / 2 \pm 2 \gamma^{\prime} \epsilon_{A}$, where $2 \epsilon_{A}=\sqrt{\delta^{2}+\Gamma_{S}^{2}}$ is a splitting between $|+\rangle$ and $|-\rangle$ states, while $\delta=2 \epsilon_{0}+U$ is a detuning between $|0\rangle$ and $|D\rangle$ states $[2,4,5]$. We also introduce the effective tunneling rates $\Gamma_{\alpha}$ to describe electron transfer between $\mathrm{QD}$ and the normal metal electrodes, $\alpha=\{L, R\}$.

When couplings to the "external" normal electrodes $\Gamma_{L(R)}$ are weak (i.e. in the sequential tunneling limit) and the transport is unidirectional (the chemical potentials $\mu_{\alpha}$ of the normal electrodes is much larger than all relevant energy scales of the system apart from the superconducting gap) the dynamics of the system is Markovian. The generalized master equation (GME) for the reduced system density operator $\rho(t)$ has a form $\dot{\rho}=\mathcal{L} \rho(t)$ where $\mathcal{L}$ is Liouvillan of the system. One can rewrite GME in terms of the jump operators $\mathcal{J}_{k}$ which describes various single electrons tunneling processes from (to) the normal electrode $L(R)$ into (from) the "proximized" QD as $\dot{\rho}=\left(\mathcal{L}_{0}+\sum_{k} \mathcal{J}_{k}\right) \rho(t) . \mathcal{L}_{0}$ is uniquely defined by the condition $\mathcal{L}_{0} \equiv \mathcal{L}-\sum_{k} \mathcal{J}_{k}$. One can define WTD describing probability distribution $w_{k, l}(\tau)$ for subsequent jumps of types $l$ and $k$ separated by time $\tau$ as [1]

$$
w_{k, l}(\tau)=\frac{\operatorname{Tr}\left[\mathcal{J}_{k} \mathrm{e}^{\mathcal{L}_{0} \tau} \mathcal{J}_{l} \rho_{0}\right]}{\operatorname{Tr}\left[\mathcal{J}_{l} \rho_{0}\right]},
$$

where $\rho_{0}$ denotes the stationary state which is a solution of $\mathcal{L} \rho_{0}=0$ and $\mathcal{L}_{0}$ describes the coherent time evolution of the proximized QD without jumps to the normal electrodes. It is worth noting that $\operatorname{Tr}\left[\mathcal{J}_{l} \rho_{0}\right]$ defines a stationary current $I_{l}$ for the type $l$ jump processes. 


\section{Results}

In order to study electron dynamics we define a reduced density matrix as a column vector $\rho=$ $\left(\rho_{00}, \rho_{\uparrow \uparrow}, \rho_{\downarrow \downarrow}, \rho_{D D}, \rho_{D 0}, \rho_{0 D}\right)^{T}$ where $\rho_{\xi \xi^{\prime}}=\left\langle\xi|\rho| \xi^{\prime}\right\rangle$ and $\xi, \xi^{\prime} \in\{0, \uparrow, \downarrow, D\}$. The diagonal elements of the density matrix $\rho_{\xi \xi}$ describes occupation probabilities of the QD while the non-diagonal elements $\rho_{D 0}$ and $\rho_{0 D}$ are known as coherences. In addition we assume the unidirectional transport (large bias voltage limit) with the only possible processes between the proximized QD and the normal electrodes. Therefore, the Liouvillian of the system takes the form

$$
\mathcal{L}=\left(\begin{array}{cccccc}
-2 \Gamma_{L} & \Gamma_{R} & \Gamma_{R} & 0 & \frac{\mathrm{i} \Gamma_{S}}{2} & -\frac{\mathrm{i} \Gamma_{S}}{2} \\
\Gamma_{L} & -\Gamma_{N} & 0 & \Gamma_{R} & 0 & 0 \\
\Gamma_{L} & 0 & -\Gamma_{N} & \Gamma_{R} & 0 & 0 \\
0 & \Gamma_{L} & \Gamma_{L} & -2 \Gamma_{R} & -\frac{\mathrm{i} \Gamma_{S}}{2} & \frac{\mathrm{i} \Gamma_{S}}{2} \\
\frac{\mathrm{i} \Gamma_{S}}{2} & 0 & 0 & -\frac{\mathrm{i} \Gamma_{S}}{2} & \Gamma_{N}^{\frac{1}{N}} & 0 \\
-\frac{\mathrm{i} \Gamma_{S}}{2} & 0 & 0 & \frac{\mathrm{i} \Gamma_{S}}{2} & 0 & \Gamma_{N}^{+}
\end{array}\right),
$$

where $\Gamma_{N}=\Gamma_{L}+\Gamma_{R}$ and $\Gamma_{N}^{ \pm} \equiv-\Gamma_{N} \pm i \delta$. We introduce jump operator $\mathcal{J}_{L e}\left(\mathcal{J}_{R e}\right)$ through the $L(R)$ junction which describes transitions from the singly occupied odd states $(|\sigma\rangle)$ to the even occupation sector $(|D\rangle$ or $|0\rangle)$. The reverse processes, which transfer an electron from the even to odd sector are described by $\mathcal{J}_{\text {Lo }}\left(\mathcal{J}_{R o}\right)$.

According to Eq. (1) one can analyse either local WTD (i.e. between subsequent jumps on the same electrode) or non-local WTD (for subsequent jumps, which occurs through different contacts). The results of calculations are shown in Fig. 1 for several values of the coupling to the right electrode $\Gamma_{R}$ and different position of the QD energy level $\epsilon_{0}$. It is worth to notice that the WTD for subsequent jumps from the same sector (e.g. $w_{L e, L e}(\tau), w_{L o, R o}(\tau)$, e.t.c. $)$ are zero.

Let's focus on $w_{L e, L o}(\tau)=w_{L e, R o}(\tau)$. After the jump $\left(J_{L o}\right.$ or $\left.J_{R o}\right)$ to the odd sector the system is in the initial single electron state, so is decoupled from the superconducting electrode. Then the next jump $\left(J_{L e}\right)$ is a Poissonian process with the rate $\Gamma_{L}$. Therefore amplitudes of the waiting times $w_{L e, L o}(\tau)=w_{L e, R o}(\tau)=$ $\Gamma_{L} \exp \left(-\Gamma_{N} \tau\right)$ decrease exponentially as a function of $\tau$ with the rate which can be tuned by $\Gamma_{R}$. On the other hand $w_{R e, L o}(\tau)=w_{R e, R o}(\tau)=\Gamma_{R} / \Gamma_{L} w_{L e, L o}(\tau)$ with the amplitude $w_{R e, L o}(\tau=0)=w_{R e, R o}(\tau=0)=\Gamma_{R}$. As one can see, all aforementioned WTDs do not depend on $\Gamma_{S}$ and $\epsilon_{0}$.

The situation changes when the system is prepared in one of the even states $|0\rangle$ or $|D\rangle$, respectively. In Fig. 1 we have plotted non-local $w_{L o, R e}(\tau)$ (thick curves) and local $w_{R o, R e}(\tau)$ (thin curves) for the electron-hole (e-h) symmetry point $\epsilon_{0}=-U / 2(\delta=0)$ and $\Gamma_{R}<\Gamma_{L}$ or $\Gamma_{R}>\Gamma_{L}$. In this case one observes oscillations with the period $T \approx \pi / \epsilon_{A}$ (i.e. inverse proportional to the splitting between $|+\rangle$ and $|-\rangle$ states). The oscillation period can be only slightly modified by $\Gamma_{L(R)}$ because $\Gamma_{S} \gg \Gamma_{L(R)}$. The non-local WTD for $\tau=0$ has a finite amplitude because the transition $\mathcal{J}_{L o}:|0\rangle \rightarrow|\sigma\rangle$ is pos-

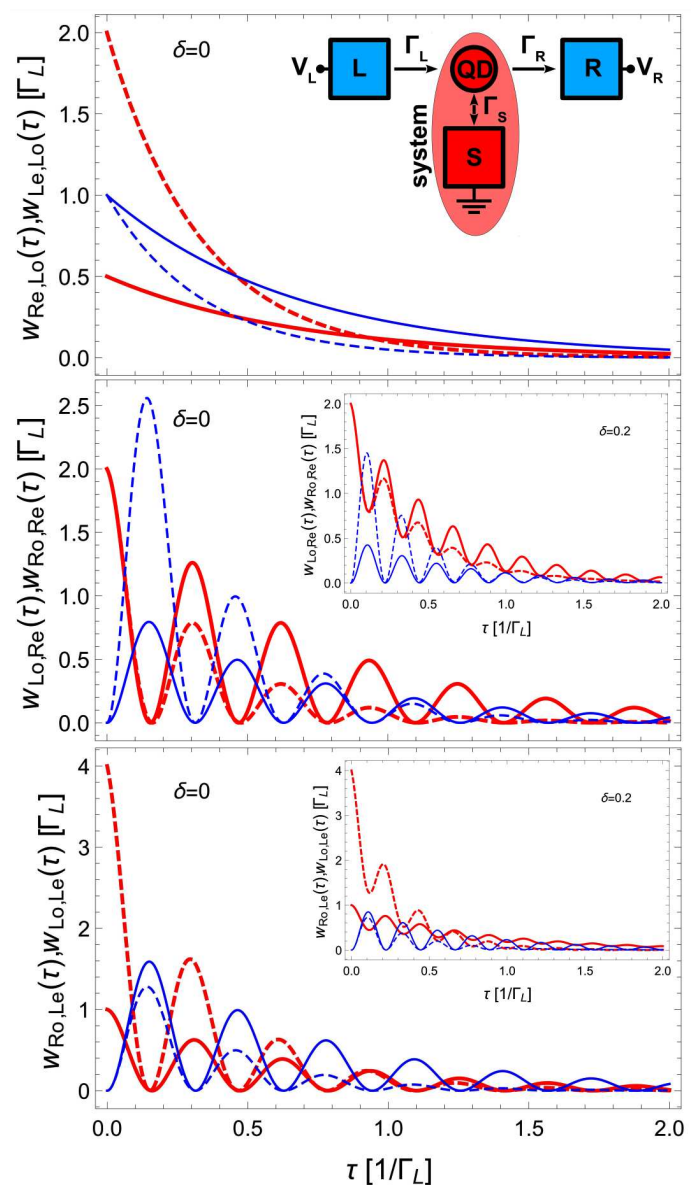

Fig. 1. Inset: Schematic view of the system, consisting of the "proximized" quantum dot $(Q D)$ weakly coupled with the left $(L)$ and right $(R)$ normal electrodes. Local (blue thin lines) and non-local (red thick lines) WTD for electron-hole symmetry point $\delta=0(\epsilon=-U / 2)$ and $\Gamma_{R}=\Gamma_{L} / 2$ (solid lines) or $\Gamma_{R}=2 \Gamma_{L}$ (dashed lines). For $w_{R e, L o}(\tau)$ and $w_{L e, L o}(\tau)$ the initial state (the state after the first jump) is $|\sigma\rangle$, for the $w_{L o(R o), R e}(\tau)$ is $|0\rangle$ while for $w_{L o(R o), L e}(\tau)$ is $|D\rangle$. Other parameters are $U=1, \Gamma_{L}=0.01, \Gamma_{S}=0.2$. Inset shows results for $\delta=0.2(\epsilon=-0.4 U)$.

sible. On the other hand the local WTD starts with 0 because first the system has to evolve to the state $|D\rangle$ due to the coherent tunneling of the Cooper pair from the superconductor to the QD. Therefore both curves for the non-local and local WTD are shifted in a phase by $\phi=T / 2$. The maximal amplitude of local $w_{R o, R e}(\tau)$ increases with $\Gamma_{R}$ while for the non-local $w_{L o, R e}(\tau)$ it seems unchanged. For both local and non-local WTD, the amplitudes of oscillations disappear faster in time as the coupling to the $R$ electrode increases. The nonlocal $w_{R o, L e}(\tau)$ (thick lines) and local $w_{L o, L e}(\tau)$ show similar behaviour, although the amplitudes are different, see Fig. 1. In the insets we have plotted results for $\epsilon_{0} \neq-U / 2(\delta \neq 0)$ i.e. away from the e-h symmetry point. Now the non-local WTD characteristics show different behaviour than local ones. It is interesting that a 


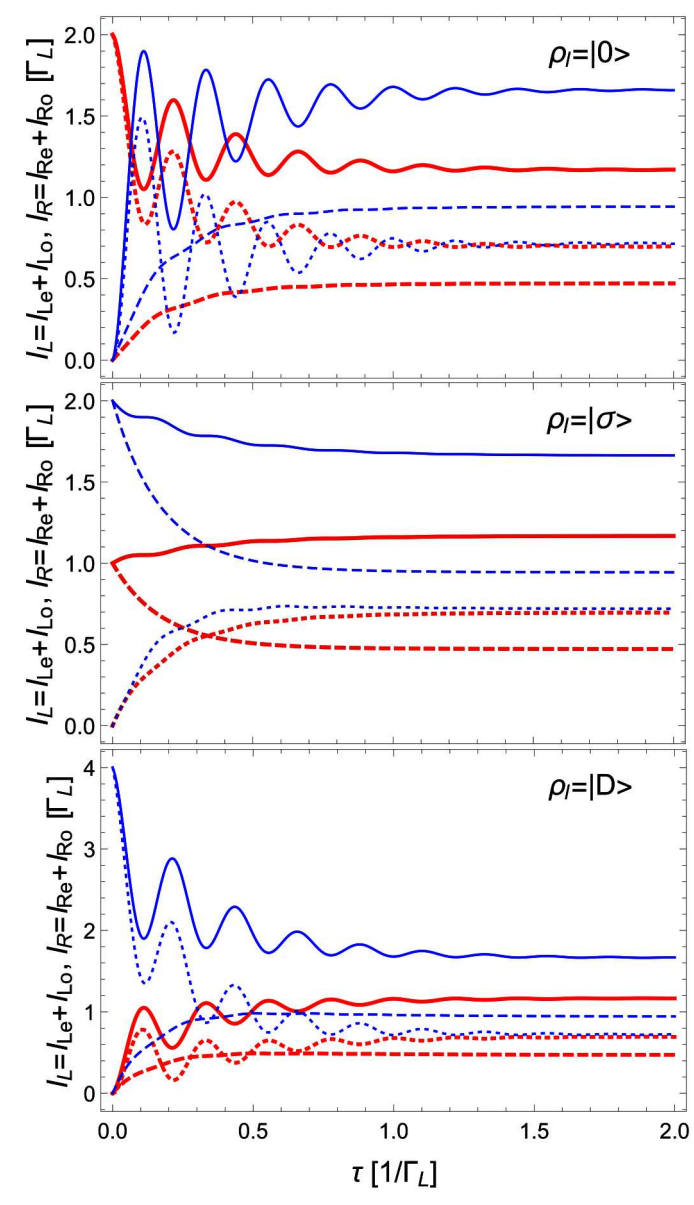

Fig. 2. Transient (relaxation) currents: $I_{L}$ (thick red line) and its components $I_{L e}$ (thick red dashed line) and $I_{L o}$ (thick red dotted line), $I_{R}$ and its components (thin blue lines) for $\Gamma_{R}=2 \Gamma_{L}$ and $\delta=0.2$.

gate voltage (i.e. a shift of the $\epsilon_{0}$ ) does not change the amplitude of the non-local WTDs for $\tau=0$ ( $c f$ results in the main figures and insets). Moreover the non-local WTDs are now non-zero functions of $\tau$.

To have some insight into microscopic processes we have calculated transient (relaxation) currents defined as $I_{k}(\tau)=\operatorname{Tr}\left[\mathcal{J}_{k} \mathrm{e}^{\mathcal{L} \tau} \rho_{l}\right]$ where $\rho_{l} \equiv\{|0\rangle,|\sigma\rangle,|D\rangle\}$. The results are shown in Fig. 2 for the total current flowing through the left (right) tunnel junction, $I_{L} \equiv I_{L e}+I_{L o}$ $\left(I_{R} \equiv I_{R e}+I_{R o}\right)$ and their components. The clearly visible oscillating currents (with the period $T=\pi / \epsilon_{A}$ which do not depend on the coupling $\left.\Gamma_{L(R)}\right)$ are activated when the system is initially in one of the even state $(|0\rangle$ or $|D\rangle)$. Because $I_{R} \neq I_{L}$ due to their phase shift one can also find transient currents flowing between QD and the superconducting electrode, $I_{S}=I_{L}-I_{R}$. It also oscillates in time and can be positive, negative or can change its sign depending on the system parameters. From the transient currents one can find dominant relaxation processes in different time scales. In the very short time scale the system relaxes from the state $|0\rangle$ mainly due to the transitions $\mathcal{J}_{L o}:|0\rangle \rightarrow|\sigma\rangle$ with the relaxation time $1 / T \sim 2 \Gamma_{L}$ while from the states $|D\rangle$ due to processes
$\mathcal{J}_{R o}:|D\rangle \rightarrow|\sigma\rangle$ with the relaxation time $1 / T \sim 2 \Gamma_{R}$. On the other hand, in the long time limit (i.e. in the stationary state) dominate processes through the $R$ junction $\mathcal{J}_{R e}:|\sigma\rangle \rightarrow|0\rangle$ and $\mathcal{J}_{R o}:|D\rangle \rightarrow|\sigma\rangle$ because $\Gamma_{R}>\Gamma_{L}$. In addition components $I_{L e}$ (due to $\mathcal{J}_{L e}:|\sigma\rangle \rightarrow|D\rangle$ ) or $I_{R e}$ (due to $\mathcal{J}_{R e}:|\sigma\rangle \rightarrow|0\rangle$ ) are always monotonically increasing function of time. In general their amplitude is different when the $\Gamma_{R} \neq \Gamma_{L}$.

When the system is initially in the single occupied state $|\sigma\rangle$ one do not see oscillations. Now at the short time limit the two processes are responsible for the relaxation, $\mathcal{J}_{R e}:|\sigma\rangle \rightarrow|0\rangle$ and $\mathcal{J}_{L e}:|\sigma\rangle \rightarrow|D\rangle$. Both with the same relaxation time $1 / T \sim \Gamma_{N}$. Their amplitude can be tuned by $\Gamma_{R}$.

\section{Conclusions}

In the multiterminal systems apart from the local WTDs one can define also non-local WTDs which show different behaviour when the system works far from the electron-hole symmetry point. The WTDs oscillate due to coherent transitions of the Cooper pairs between QD and the superconductor. These oscillation are also clearly visible in transient currents characteristics. In addition the transient currents analysis shows a dominant microscopic relaxation processes on both the short and the long time scales.

\section{Acknowledgments}

The research was financed by National Science Centre, Poland - project numbers 2016/21/B/ST3/02160 (BRB, GM) and DEC-2014/13/B/ST3/04451 (KIW, TD).

\section{References}

[1] T. Brandes, Ann. Phys. (Berlin) 17, 477 (2008).

[2] L. Rajabi, Ch. Pöltl, M. Governale, Phys. Rev. Lett.111, 067002 (2013).

[3] J. Gramich, A. Baumgartner, C. Schönenberger, arXiv:1612.01201v1.

[4] K. I. Wysokiński, J. Phys.: Condens. Matter 24, 335303 (2012).

[5] A. Braggio, M. Governale, M. G. Pala, J. König, Solid State Communications 151, 155 (2011). 